\title{
Surgical Outcomes of Patients with Sacroiliac Joint Pain: An Analysis of Patients with Poor Results Regarding Activities of Daily Living
}

\author{
Daisuke Kurosawa $^{1)}$, Eiichi Murakami ${ }^{1)}$, Toshimi Aizawa ${ }^{2)}$ and Takashi Watanabe ${ }^{3)}$ \\ 1) Department of Orthopaedic Surgery/Low Back Pain and Sacroiliac Joint Center, JCHO Sendai Hospital, Sendai, Japan \\ 2) Department of Orthopaedic Surgery, Tohoku University School of Medicine, Sendai, Japan \\ 3) Department of Hospital Medicine, JCHO Sendai Hospital, Sendai, Japan
}

\begin{abstract}
:
Introduction: Sacroiliac joint (SIJ) arthrodesis is the last resort for patients with severe SIJ pain. However, this technique does not always provide good outcomes regarding activities of daily living (ADL). This study aims to reveal the preoperative clinical features associated with poor outcomes of SIJ arthrodesis.

Methods: Twenty-six consecutive patients who underwent SIJ arthrodesis between 2009 and 2018 were evaluated. Goodoutcome was defined as $\geq 30 \%$ improvement in ADL, quantified by the Roland-Morris Disability Questionnaire (RDQ). The good-outcome group (17 patients; 10 men and 7 women, $42.5 \pm 8.4$ years old) and the poor-outcome group (9 patients; 1 man and 8 women, $47.0 \pm 17.9$ years old) were compared to identify the preoperative clinical features of poor surgical outcomes.

Results: No significant differences were observed between the two groups regarding age, preoperative RDQ score, time between onset and the confirmed diagnosis of SIJ pain, and the time between diagnosis and surgical treatment. The following preoperative clinical features associated with poor surgical outcomes were identified: female sex, pain in multiple regions, walking with a cane, and the use of a wheelchair before surgery $(\mathrm{P}<0.05)$.

Conclusions: The present study demonstrated that poor postoperative outcomes in patients with severe SIJ pain were associated with the following preoperative clinical features: female sex, pain in multiple regions, walking with a cane, or use of a wheelchair. Ample attention is warranted in patients with such features who are indicated for surgical treatments.
\end{abstract}

Keywords:

Sacroiliac joint, arthrodesis, poor results, activities of daily living, clinical features

Spine Surg Relat Res 2021; 5(3): 189-195 dx.doi.org/10.22603/ssrr.2020-0214

\section{Introduction}

Sacroiliac joint (SIJ) dysfunction is recognized as one of the major causes of low back and buttock pain ${ }^{1,2)}$. Conservative treatments such as SIJ injections, radiofrequency neurotomy, pelvic belt, and various types of physical therapy, including mobilization and manipulation ${ }^{3)}$, are effective in patients with SIJ pain. However, SIJ arthrodesis is the last resort for patients when such conservative treatments cannot significantly reduce pain.

Surgical procedures for SIJ have been developing since the $1920 \mathrm{~s}^{4)}$. However, the number of patients that underwent surgery was limited. Recently, several new products for SIJ surgery have been developed ${ }^{5-7)}$, and many surgeons have realized that dealing with SIJ problems can become part of their duties. There are many reports of excellent surgical outcomes achieved with new products ${ }^{6-8)}$.

Conversely, previously reported surgical outcomes were not always satisfactory ${ }^{5,9-12)}$. Several factors, including misdiagnosis, pseudoarthrosis, pathological conditions other than joint instability, and psychosomatic disorders, have been considered as the reasons why the surgical procedure failed to relieve SIJ pain and fully improve the functional status. However, preoperative features that were associated with poor surgical results still remained elusive. Thus, this study aims to clarify the preoperative clinical features that are as-

Corresponding author: Daisuke Kurosawa, d-kurorin@m3.dion.ne.jp

Received: November 25, 2020, Accepted: December 15, 2020, Advance Publication: January 21, 2021

Copyright (C) 2021 The Japanese Society for Spine Surgery and Related Research 
Table 1. Surgical Procedures.

\begin{tabular}{lc}
\hline \multicolumn{1}{c}{ Surgical approach } & Number of fixed joints \\
\hline Anterior alone & 11 \\
Combined anterior and posterior & 3 \\
Bilateral anterior and pubic symphysis fixation (pelvic ring fixation) & 2 (one patient) \\
Posterior alone & 13 \\
Lateral alone & 2 \\
\hline
\end{tabular}
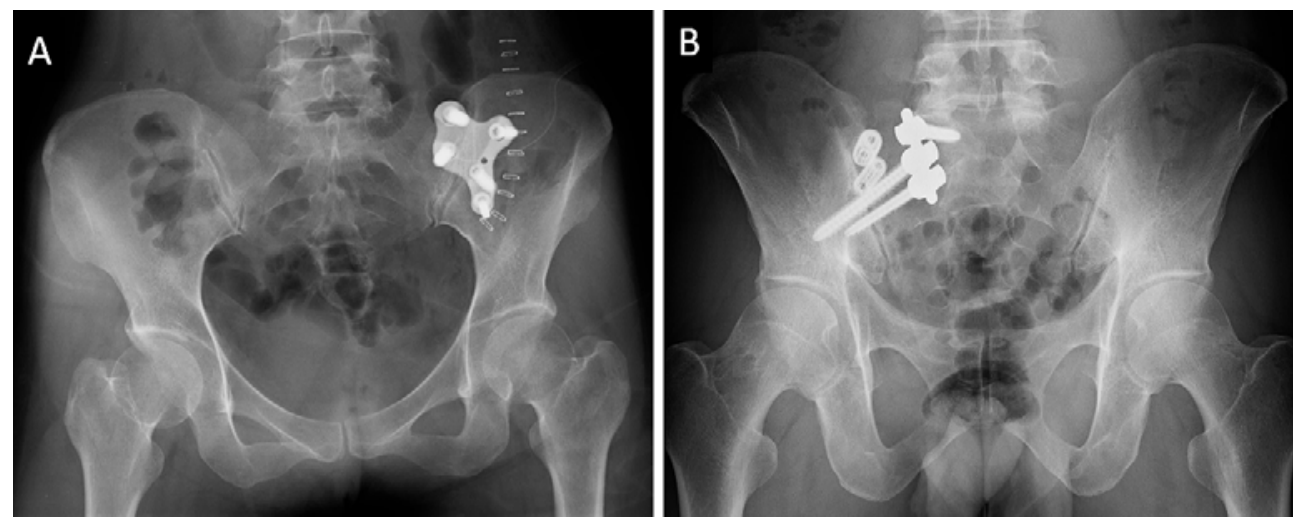

Figure 1. Techniques of SIJ arthrodesis.

A. Anterior SIJ arthrodesis using plate and screws.

B. Posterior SIJ arthrodesis using an S1 pedicle screw and two S2 alar-iliac screws and cylinder cages.

sociated with poor outcomes of SIJ arthrodesis (particularly those regarding activities of daily living (ADL)), based on the comparative analyses of preoperative conditions, symptoms, background, and image-based findings between the good- and poor-outcome groups.

\section{Materials and Methods}

\section{Study design}

This case-control study compared two surgical outcome groups categorized on the basis of their ADL as good- and poor-outcome groups, to identify preoperative conditions which could relate to poor outcomes after SIJ arthrodesis.

This study was approved by the Institutional Review Board of our hospital. Written informed consent was obtained from patients for the use of their data.

\section{Patients}

Thirty patients with SIJ pain underwent SIJ arthrodesis between April 2009 and March 2018 in a single hospital, and follow-up of these cases was scheduled until March 2019. Four patients did not continue to follow-up; thus, 26 of these patients (11 men and 15 women) were evaluated. The mean age at surgery was 44 (range, 26-79) years. A total of 31 joints underwent SIJ arthrodesis (Table 1, Fig. 1), and the mean follow-up time was $46.7 \pm 33.0$ months.

All patients identified the posterior superior iliac spine (PSIS) as the main location of pain, using their index finger (one-finger test) ${ }^{13)}$, and scored more than 4 points in the SIJ scoring system ${ }^{14)}$ (Table 2). The scoring system consists of six items, namely, one-finger test, groin pain, pain while sitting on a chair, SIJ shear test, tenderness of the PSIS, and tenderness of the sacrotuberous ligament. The sum score range is from 0 to 9 points. These patients were considered to have SIJ-related symptoms. The definitive diagnosis of SIJ pain was confirmed by $\geq 70 \%$ pain relief in the region of the SIJ after SIJ fluoroscopic guided injection ${ }^{14,15}$. Injection efficacy was evaluated using the pain relief scale ${ }^{16)}$. All patients were asked to report the postinjection pain intensity, assuming that the pain score before injection was 10 . The remaining pain was recorded 15 min postinjection. Pain improvement $\geq 70 \%$ was assumed if the patients reported a postinjection remaining pain intensity score of $\leq 3$. We considered that the patient had an isolated condition with only SIJ pain when any other injection, except for those specific to SIJ, was not effective. We excluded patients with a history of infection, tumors in the lumbar and pelvic regions, recent lumbar spine and pelvic fractures, and obvious ankylosing spondylitis. All patients had a history of other injections, including selective nerve root infiltration and/or lumbar disc block, and those injections showed a negative response.

\section{Indications for surgical treatment and definition of good postoperative outcomes}

The indication for SIJ arthrodesis was insufficient responsiveness to conservative treatments for $\geq 6$ months, difficulty 
Table 2. Scoring System for Sacroiliac Joint-related Symptoms.

\begin{tabular}{lc}
\hline \multicolumn{1}{c}{ Item } & Score \\
\hline One-finger test & 3 \\
Groin pain & 2 \\
Pain while sitting on a chair & 1 \\
Sacroiliac joint shear test & 1 \\
Tenderness of PSIS & 1 \\
Tenderness of STL & 1 \\
\hline \multicolumn{1}{c}{ Total } & 9 \\
\hline
\end{tabular}

PSIS: posterior superior iliac spine; STL: sacrotuberous ligament

in working, and marked restrictions in the ADL due to recurrence of severe SIJ pain, even after undergoing repeated injections and substantial physical therapies as inpatients ${ }^{17)}$. The ADL of patients was assessed pre- and postoperatively using the Roland-Morris Disability Questionnaire (RDQ). The outcome was defined as good when an improvement $\geq 30 \%$ was obtained in the $\mathrm{RDQ}^{18)}$.

According to the above definition, the good-outcome group included 17 patients (10 men and 7 women) showing an improvement $\geq 30 \%$ in the RDQ score; the poor-outcome group included 9 patients ( 1 man and 8 women) showing an improvement $\leq 30 \%$ in the RDQ score (Fig. 2).

\section{Data collection}

The good- and poor-outcome groups were compared to identify the preoperative clinical features of poor surgical outcomes. The following items were evaluated in this study: pre- and postoperative pain intensity (visual analog scale, VAS) and the RDQ score, age, sex, time between onset and confirmed definite diagnosis of SIJ pain, time between diagnosis and surgical treatment, SIJ pain existed unilaterally or bilaterally, presence or absence of pain in multiple regions other than the $\mathrm{SIJ}^{5,9)}$, sitting tolerance [time while patients can tolerate pain when sitting on a chair with no back rest ( $<5 \mathrm{~min})$ ], walking with a cane, use of a wheelchair, pain in the supine position, pain while lying on the painful side, rest pain, night pain, pain/numbness in the lower limbs, any accident which induced SIJ pain (such as a traffic accident, twisting, lifting a heavy weight, falling onto the buttocks), as well as combined or past medical problems (lumbar, cervical spine, hip, and mental disorders), preoperative CT image-based findings (osteophytes, vacuum phenomena ${ }^{19}$, or erosion with subchondral sclerosis ${ }^{20)}$ in the SIJ), and postoperative plain radiographs and/or CT image-based analysis on whether screw loosening was detected at $\geq 1$ year after surgery. Data regarding physical statuses were checked just before surgery.

\section{Statistical analyses}

Clinical features associated with poor surgical outcomes were identified by using univariate analysis to compare between the two groups. The Kruskal-Wallis test was used to

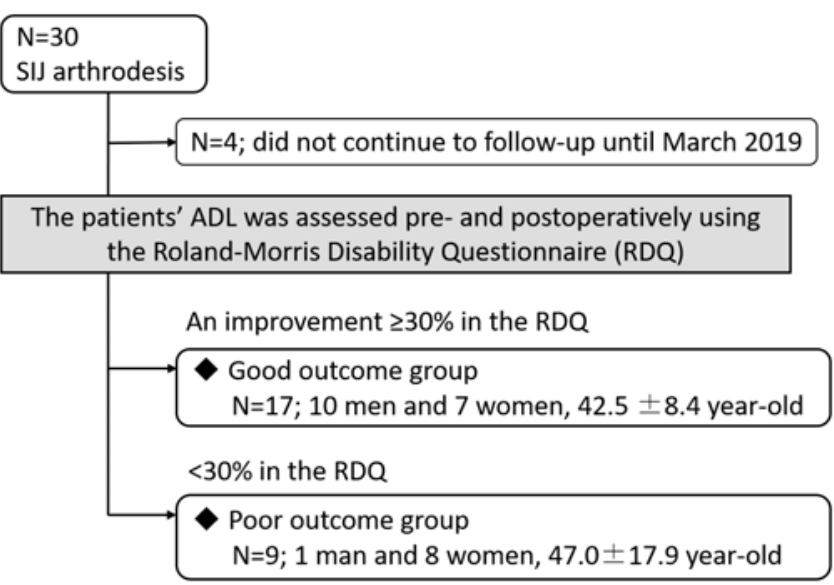

Figure 2. Flow chart to determine the two types of surgical outcome groups.

analyze VAS, the RDQ score, age, time between onset and the definite diagnosis of SIJ pain, and time between confirmed diagnosis and surgical treatment. Fisher's exact test was used to analyze other factors. A result with a P-value $<0.05$ was considered to be statistically significant.

\section{Results}

\section{Univariate analysis to identify the preoperative clinical fea- tures for poor results}

No significant differences were observed between the two groups regarding age, preoperative RDQ score, time between onset and the confirmed diagnosis of SIJ pain, and time between diagnosis and surgical treatment. In the pooroutcome group, the pre- and postoperative VAS were significantly higher than in the good-outcome group $(\mathrm{P}<0.05)$ (Table 3). Univariate analysis revealed four items that were significantly associated with poor surgical outcomes $(\mathrm{P}<0.05)$, namely, female sex, pain in multiple regions, walking with a cane, and the use of a wheelchair. The pre- and postoperative image-based findings showed no significant differences between the good- and poor-outcome groups (Table 4), although one 79-year-old female patient, who had right SIJ pain alone and walked with a cane, showed implant loosening.

\section{Case presentation}

A 52-year-old woman reported pain in the left buttock, 2 years prior, mainly after jumping at a rock concert. She complained of pain in multiple regions including the lower back, buttock, left groin, left knee, and left ankle joints. Her most severe pain area was the left SIJ. This pain originated from the left SIJ and was confirmed by SIJ injections. The patient achieved $\geq 70 \%$ pain relief in her left SIJ after an SIJ injection of $2 \mathrm{ml}$ of $1 \%$ lidocaine under fluoroscopy guidance. However, the efficacy using SIJ injection was temporary. She needed a cane while walking and used a wheelchair to go further. Analgesic drugs, including NSAIDs, pre- 
Table 3. Results of Univariate Analysis.

\begin{tabular}{lccc}
\hline & \multicolumn{2}{c}{ Improvement of ADL } & \multirow{2}{*}{ P } \\
\cline { 2 - 3 } & Good $(\mathrm{N}=17)$ & Poor $(\mathrm{N}=9)$ & \\
\hline Preoperative VAS (0-100 mm) & $86.8 \pm 8.9$ & $93.0 \pm 7.7$ & $0.03^{*}$ \\
Preoperative RDQ (0-24 points) & $18.9 \pm 4.6$ & $18.6 \pm 2.2$ & 0.27 \\
Postoperative VAS (0-100 mm) & $24.6 \pm 22.8$ & $57.4 \pm 26.9$ & $0.003^{*}$ \\
Postoperative RDQ (0-24 points) & $4 \pm 4.1$ & $18.2 \pm 2.8$ & $<0.001^{*}$ \\
Age & $42.5 \pm 8.4$ & $47.0 \pm 17.9$ & 0.81 \\
Time between onset and confirmed diagnosis of SIJ pain $(\mathrm{M})$ & $16.0 \pm 22.3$ & $10.4 \pm 12.9$ & 0.55 \\
Time between diagnosis and surgical treatment (M) & $27.9 \pm 25.2$ & $34.3 \pm 26.8$ & 0.34 \\
\hline
\end{tabular}

$* p<0.05$

ADL: Activities of Daily Living; VAS: visual analog scale; RDQ: Roland-Morris Disability Questionnaire; SIJ: sacroiliac joint; M: month

Table 4. Results of Univariate Analysis Regarding Preoperative Clinical Features.

\begin{tabular}{|c|c|c|c|}
\hline & \multicolumn{2}{|c|}{ Improvement of $\mathrm{ADL}$} & \multirow{2}{*}{$\mathrm{P}$} \\
\hline & Good $(\mathrm{N}=17)$ & Poor $(\mathrm{N}=9)$ & \\
\hline Sex: Female (\%) & $7(41.2 \%)$ & $8(82.0 \%)$ & $0.04 *$ \\
\hline Bilateral SIJ pain & $4(23.5 \%)$ & $5(55.6 \%)$ & 0.19 \\
\hline Pain in multiple regions & $0(0 \%)$ & $3(33.3 \%)$ & $0.03 *$ \\
\hline Sitting tolerance ( $<5$ minutes) & $10(58.8 \%)$ & $3(33.3 \%)$ & 0.41 \\
\hline Walking with a cane & $7(41.2 \%)$ & $8(88.9 \%)$ & $0.04 *$ \\
\hline Using a wheelchair & $3(17.6 \%)$ & $6(66.7 \%)$ & $0.03 *$ \\
\hline Pain in the supine position & $11(64.7 \%)$ & $5(55.6 \%)$ & 0.69 \\
\hline Pain while lying on the painful side & $9(52.9 \%)$ & $5(55.6 \%)$ & 1.00 \\
\hline Rest pain & $7(41.2 \%)$ & $6(66.7 \%)$ & 0.41 \\
\hline Night pain & $7(41.2 \%)$ & $6(66.7 \%)$ & 0.41 \\
\hline Pain in the lower extremities & $8(47.1 \%)$ & $6(66.7 \%)$ & 0.43 \\
\hline Leg numbness & $11(64.7 \%)$ & $7(77.8 \%)$ & 0.67 \\
\hline Any accident which induced SIJ pain & $12(70.6 \%)$ & $4(44.4 \%)$ & 0.23 \\
\hline \multicolumn{4}{|l|}{ Combined or past medical problems } \\
\hline Lumbar disorders & $5(29.4 \%)$ & $2(22.2 \%)$ & 1.00 \\
\hline Cervical disorders & $1(5.9 \%)$ & $0(0 \%)$ & 1.00 \\
\hline Hip disorders & $1(5.9 \%)$ & $1(11.1 \%)$ & 1.00 \\
\hline Mental disorders & $3(17.6 \%)$ & $0(0 \%)$ & 0.53 \\
\hline CT findings of the SIJ (osteophytes and vacuum phenomena/erosion with subchondral sclerosis) & $6(35.3 \%)$ & $6(66.7 \%)$ & 0.22 \\
\hline Implant loosening & $0(0 \%)$ & $1(9 \%)$ & 0.35 \\
\hline
\end{tabular}

gabalin, and amitriptyline, only provided mild pain relief. After 2-year conservative treatments, we performed arthrodesis on the left SIJ using triangular implants via the lateral approach. Postoperatively, her left SIJ pain dramatically decreased. VAS changed from $90 \mathrm{~mm}$ to $15 \mathrm{~mm}$, and she was satisfied with the surgical outcome. However, her ADL improvement was below our expectations; the RDQ score was 15 points preoperatively and did not change postoperatively. Except for the left SIJ, other multiple regional pain persisted, and she continued to use a cane and a wheelchair (Fig. 3).

\section{Discussion}

Excellent surgical outcomes of SIJ arthrodesis have been achieved with new products ${ }^{6,8}$. Previous studies on SIJ arthrodesis have shown that approximately $75 \%$ of patients obtained good results ${ }^{9,12)}$. Although the remaining $25 \%$ of patients indicated poor surgical outcomes, little information exist about those patients in the literature ${ }^{9}$. Risk factors for poor surgical results have not been revealed. Therefore, in this study, we focused on those patients in order to clarify the preoperative clinical features associated with poor surgical results.

In this study, we used the improvement in ADL, not pain intensity, to assess the outcome of SIJ arthrodesis. Pre- and 

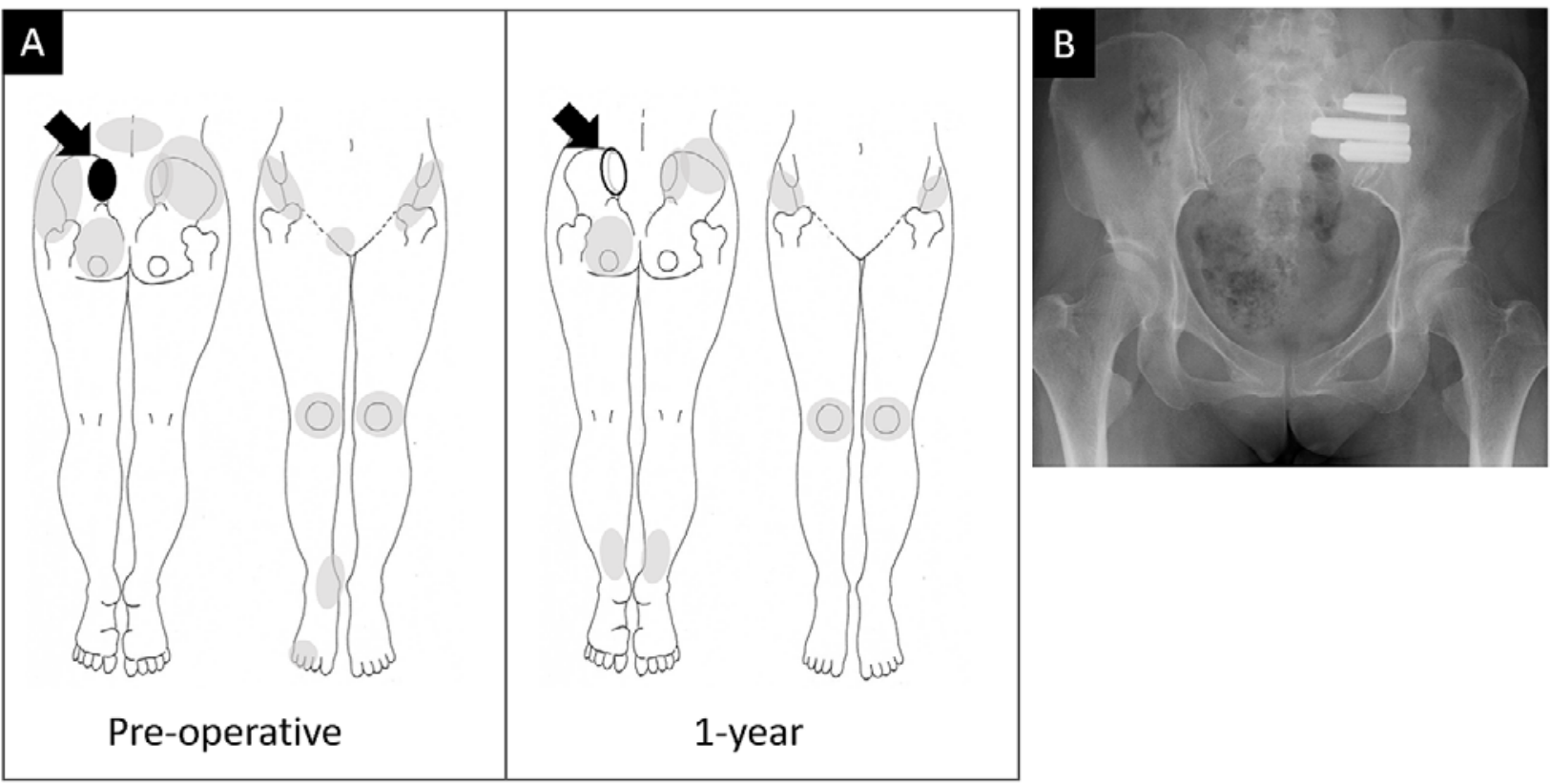

Figure 3. A 52-year-old woman who reported pain in the left buttock, mainly after jumping at a rock concert.

A. Pre- and postoperative pain area. Pain in her left SIJ side improved. However, pain in multiple regions persisted, and she continued to use a cane and wheelchair.

B. Plain radiographs 1 year after surgery.

postoperative pain intensity are important factors for evaluating the state of the patients; however, VAS may change depending on the day. Therefore, Kibsgård evaluated VAS repeatedly in different phases ${ }^{9)}$. VAS is a subjective assessment and depends on the pain sensitivity of the individual patient. ADL assessment based on the RDQ is more objective than VAS. Therefore, we adopted the RDQ to evaluate the outcome of SIJ arthrodesis in this study.

The instability of SIJ is the most common cause of SIJ pain. Therefore, SIJ arthrodesis is indicated for patients with severe SIJ pain. The surgical outcome would be poor if SIJ arthrodesis resulted in incomplete bone union or loosening of implants. In this study, implant loosening was observed in only one patient. In addition, bone union was not evaluated due to insufficient follow-ups on several patients. Therefore, it was not possible to compare the rate of complete bone union and the differences in the ADL between the groups.

This study revealed that preoperative clinical features, such as female sex, pain in multiple regions, walking with a cane, and the use of a wheelchair before surgery, were significantly associated with poor surgical outcomes related to ADL. There were no differences between the good- and poor-outcome groups regarding the time between the onset of symptoms and the definite diagnosis of SIJ and time between diagnosis and surgical treatment.

Gender differences in the surgical management of lumbar degenerative diseases have also been reported ${ }^{21)}$. In general, women undergoing surgery for the lumbar degenerative disease had worse absolute postoperative scores. It was suggested that women present with or are referred for assess- ment later in the course of their diseases, leading to an inferior clinical status at the time of surgery ${ }^{22-24}$. Several researches also reported differences in pain sensitivity between men and women ${ }^{25}$. More particularly, focusing on the anatomy of the SIJ, women have a larger range of motion of the SIJ than men $^{26}$. In a previous study ${ }^{17}$, both restricted hip range of motion (ROM) and reduction of walking stride after SIJ arthrodesis were observed, indicating that the hip ROM might be compounded by the hip joint itself and the SIJ. Restricted hip ROM may lead to difficulties in sitting with the legs crossed, putting on or taking off socks, squatting down and taking things off, and walking quickly, which could cause poor outcomes related to ADL. Particularly in women, fixed SIJ might affect the restriction of hip ROM more than in men and could affect household chores, leading to lower ADL.

Pain in multiple regions was a significant preoperative clinical feature of poor surgical outcomes. Fuchs also indicated that patients having multiple pain locations in the entire body, in addition to the SIJ region, preoperatively showed significantly worse surgical results comparted to patients with SIJ pain alone ${ }^{5}$. Systemic diseases, such as spondyloarthritis, psychosomatic disorders, or other unknown pathomechanisms, could be included as causes of pain in multiple regions. However, diagnosing patients with SIJ pain associated with systemic diseases can be a challenging task. Before a definitive diagnosis of the systemic disease, patients may undergo SIJ arthrodesis since they would become unemployed, and their social activity could decline due to severe SIJ pain.

Preoperative RDQ scores showed no significant difference 
between the good- and poor-outcome groups. Regrettably, the use of canes or wheelchairs was not part of the RDQ and, therefore, was not reflected in the RDQ scores. The patients who used a cane or wheelchair preoperatively indicated that they suffered from more severe SIJ pain. In addition, their muscles had become weaker because they used those support items. The severity of preoperative condition and muscle dysfunction may be related to their insufficient improvement of ADL.

This study has a few limitations. This study involved a relatively small number of patients because patients with SIJ pain who underwent surgical treatment are very rare, which may not be sufficient to evaluate the risk factors associated with poor surgical outcomes. In addition, we have modified our surgical technique during the last 10 years. Therefore, we have not been able to know how surgical methods have systematically influenced the outcomes. Thus, study groups that have performed many SIJ arthrodesis with a single type of instrument would be expected to further investigate risk factors associated with poor surgical outcomes.

This study demonstrated that poor postoperative outcomes in patients with severe SIJ pain were associated with the following preoperative clinical features: female sex, pain in multiple regions, walking with a cane, or use of a wheelchair. Further studies are needed to classify the causes of SIJ pain, determine the precise timing of SIJ arthrodesis, or identify those patients in whom surgery is contraindicated.

Conflicts of Interest: The authors declare that there are no relevant conflicts of interest.

Ethical Approval: This study was approved by the Institutional Review Board of JCHO Sendai Hospital (No. 201823). Patients provided written informed consent for the use of their data in this study.

Author Contributions: Daisuke Kurosawa: conceptualization, methodology, investigation, and writing of the original draft. Eiichi Murakami: conceptualization, investigation, supervision, and writing of the original draft. Toshimi Aizawa: writing/review and editing and supervision. Takashi Watanabe: methodology and software.

\section{References}

1. Bernard TN, Kirkaldy-Wills WH. Recognizing specific characteristics of nonspecific low back pain. Clin Orthop Relat Res. 1987; 217:266-80.

2. Schwarzer AC, Aprill CN, Bogduk N. The sacroiliac joint in chronic low back pain. Spine (Phila Pa 1976). 1995;20(1):31-7.

3. Bernard TN, Classidy JD. The Adult Spine: Principles and Practice. Philadelphia: Lippincott-Raven Publishers; 1997. Chapter 109, The sacroiliac joint syndrome. Pathophysiology, diagnosis and management; p. 2343-63.

4. Smith-Petersen MN, Rogers WA. End-result study of arthrodesis of the sacro-iliac joint for arthritis-traumatic and non-traumatic. J Bone Joint Surg. 1926;8-A:118.

5. Fuchs V, Ruhl B. Distraction arthrodesis of the sacroiliac joint: 2- year results of a descriptive prospective multi-center cohort study in 171 patients. Eur Spine J. 2018;27(1):194-204.

6. Dengler J, Kools D, Pflugmacher R, et al. Randomized trial of sacroiliac joint arthrodesis compared with conservative management for chronic low back pain attributed to the sacroiliac joint. J Bone Joint Surg Am. 2019;101(5):400-11.

7. Miller LE, Block JE. Minimally invasive arthrodesis for chronic sacroiliac joint dysfunction using the simmetry SI joint fusion system. Med Devices (Auckl). 2014;7:125-30.

8. Rainov NG, Schneiderhan R, Heidecke V. Triangular titanium implants for sacroiliac joint fusion. Eur Spine J. 2019;28(4):727-34.

9. Kibsgård TJ, Røise O, Stuge B. Pelvic joint fusion in patients with severe pelvic girdle pain - a prospective single-subject research design study. BMC Musculoskelet Disord. 2014;15:85.

10. Nyström B, Gregebo B, Taube A, et al. Clinical outcome following anterior arthrodesis in patients with presumed sacroiliac joint pain. Scand J Pain. 2017;17:22-9.

11. Buchowski JM, Kebaish KM, Sinkov V, et al. Functional and radiographic outcome of sacroiliac arthrodesis for the disorders of the sacroiliac joint. Spine J. 2005;5(5):520-9.

12. Zaidi HA, Montoure AJ, Dickman CA. Surgical and clinical efficacy of sacroiliac joint fusion: a systematic review of the literature. J Neurosurg Spine. 2015;23(1):59-66.

13. Murakami E, Aizawa T, Noguchi K, et al. Diagram specific to sacroiliac joint pain site indicated by one-finger test. J Orthop Sci. 2008;13(6):492-7.

14. Kurosawa D, Murakami E, Ozawa H, et al. A diagnostic scoring system for sacroiliac joint pain originating from the posterior ligament. Pain Med. 2017;18(2):228-38.

15. Murakami E, Kurosawa D, Aizawa T. Treatment strategy for sacroiliac joint-related pain at the posterior superior iliac spine. Clin Neurol Neurosurg. 2018;165:43-6.

16. Lee JJ, Lee KM, Kim JE, et al. Pain relief scale is more highly correlated with numerical rating scale than with visual analogue scale in chronic pain patients. Pain Physician. 2015;18(2):E195200.

17. Murakami E, Kurosawa D, Aizawa T. Sacroiliac joint arthrodesis for chronic sacroiliac joint pain: an anterior approach and clinical outcomes with a minimum 5-year follow-up. J Neurosurg Spine. 2018;29(3):279-85

18. Ostelo RW, Deyo RA, Stratford P, et al. Interpreting change scores for pain and functional status in low back pain: towards international consensus regarding minimal important change. Spine (Phila Pa 1976). 2008;33(1):90-4.

19. Bäcklund J, Clewett Dahl E, Skorpil M. Is CT indicated in diagnosing sacroiliac joint degeneration? Clin Radiol. 2017;72(8):693.

20. Sieper J, Braun J. Clinician's manual on axial spondyloarthritis. Springer healthcare, London; 2014. $57 \mathrm{p}$

21. MacLean MA, Touchette CJ, Han JH, et al. Gender differences in the surgical management of lumbar degenerative disease: a scoping review. J Neurosurg Spine. 2020;31:1-18.

22. Pochon L, Kleinstück FS, Porchet F, et al. Influence of gender on patient-oriented outcomes in spine surgery. Eur Spine J. 2016;25 (1):235-46.

23. Siccoli A, Staartjes VE, de Wispelaere MP, et al. Gender differences in degenerative spine surgery: do female patients really fare worse? Eur Spine J. 2018;27(10):2427-35.

24. Triebel J, Snellman G, Sandén B, et al. Women do not fare worse than men after lumbar fusion surgery: Two-year follow-up results from 4,780 prospectively collected patients in the Swedish National Spine Register with lumbar degenerative disc disease and chronic low back pain. Spine J. 2017;17(5):656-62. 
25. Soetanto AL, Chung JW, Wong TK. Are there gender differences in pain perception? J Neurosci Nurs. 2006;38(3):172-6.

26. Joukar A, Shah A, Kiapour A, et al. Sex specific sacroiliac joint biomechanics during standing upright: A finite element study. Spine (Phila Pa 1976). 2018;43(18):E1053-60.
Spine Surgery and Related Research is an Open Access journal distributed under the Creative Commons Attribution-NonCommercial-NoDerivatives 4.0 International License. To view the details of this license, please visit (https://creativeco mmons.org/licenses/by-nc-nd/4.0/). 